\title{
Article \\ Predicting the Non-Deterministic Response of a Micro-Scale Mechanical Model Using Generative Adversarial Networks
}

\author{
Albert Argilaga ${ }^{1}\left(\mathbb{D}\right.$ and Duanyang Zhuang ${ }^{1,2, *(1)}$ \\ 1 MOE Key Laboratory of Soft Soils and Geoenvironmental Engineering, Zhejiang University, \\ Hangzhou 310058, China; argilaga@zju.edu.cn \\ 2 Center for Hypergravity Experiment and Interdisciplinary Research, Zhejiang University, \\ Hangzhou 310058, China \\ * Correspondence: zhuangdy@zju.edu.cn
}

Citation: Argilaga, A.; Zhuang, D. Predicting the Non-Deterministic Response of a Micro-Scale Model Using Generative Adversarial Networks. Materials 2022, 15, 965. https://doi.org/10.3390/ ma15030965

Academic Editors: Ivano Benedetti, Fabrice Barbe and Antonio Caggiano

Received: 30 December 2021

Accepted: 23 January 2022

Published: 26 January 2022

Publisher's Note: MDPI stays neutral with regard to jurisdictional claims in published maps and institutional affiliations.

Copyright: () 2022 by the authors. Licensee MDPI, Basel, Switzerland. This article is an open access article distributed under the terms and conditions of the Creative Commons Attribution (CC BY) license (https:/ / creativecommons.org/licenses/by/ $4.0 /)$.

\begin{abstract}
Recent improvements in micro-scale material descriptions allow to build increasingly refined multiscale models in geomechanics. This often comes at the expense of computational cost which can eventually become prohibitive. Among other characteristics, the non-determinism of a micro-scale response makes its replacement by a surrogate particularly challenging. Machine Learning (ML) is a promising technique to substitute physics-based models, nevertheless existing ML algorithms for the prediction of material response do not integrate non-determinism in the learning process. Is it possible to use the numerical output of the latest micro-scale descriptions to train a ML algorithm that will then provide a response at a much lower computational cost? A series of ML algorithms with different levels of depth and supervision are trained using a datadriven approach. Gaussian Process Regression (GPR), Self-Organizing Maps (SOM) and Generative Adversarial Networks (GANs) are tested and the latter retained because of its superior results. A modified GANs with lower network depth showed good performance in the generation of failure probability maps, with good reproduction of the non-deterministic micro-scale response. The trained generator can be incorporated into existing multiscale models allowing to, at least partially, bypass the costly micro-scale computations.
\end{abstract}

Keywords: micro-scale; constitutive law; asymptotic homogenization; Gaussian Process Regression; Self-Organizing Map; Generative Adversarial Networks; machine learning

\section{Introduction}

Auriault [1] shows that the bulk constitutive response of materials is often determined by their configuration at the micro-scale. In numerical modelling, identifying the cases that need a micro-scale description is important to advance towards more efficient and accurate multiscale models [2]. Some examples of materials with an important micro-structural contribution to the bulk response are listed: masonry structures [3,4], coal reservoirs for methane production [5-7], shale rocks [8,9] as well as other rocks [10] and brittle porous materials governing seismic events [11].

In multiscale modelling the constitutive material response is not phenomenologically defined beforehand, instead, at each loading or strain increment the material response is obtained from an underlying numerical model describing the micro-scale e.g., [12-18]. This approach makes the integration of material points much more costly, with the computational cost often becoming a limiting factor. One of the existing techniques to upscale the micro-scale behaviour to the macro-scale is through asymptotic homogenization [19-23], this technique allows to obtain an equivalent description of the micro-scale in a generic cell and use it as a material constitutive relation e.g., Auriault [24], Argilaga et al. [25]. The constitutive relation can be calculated once at the beginning of the multiscale simulation, or updated at given intervals depending on material evolution allowing to avoid the costly 
material point integrations. Asymptotic homogenization is only efficient in problems without significant material evolution. In a more general case, real time updates of the material constitutive response are required thus diluting computational economy.

Machine Learning (ML) models are an alternative to the costly micro-scale calculations. Trained ML can computationally advantage classical physics-based numerical methods in several orders of magnitude [26-28]. In the geomechanics and materials fields AI (Artificial Intelligence) and ML have shown different levels of success in multiscale problems [29,30], material constitutive modelling [31-34] as well as in the study of composite in both forward and inverse design approaches [35-37]. Some recent applications of AI in the macro modelling of geotechnical problems include: natural hazard prediction and mitigation [38], determination of driven piles bearing capacity in sands using ANN [39], advanced ML techniques [40] and AI systems optimized by evolutionary computation [41], determination of slope stability with ANN [42], among others.

In some cases materials present loss of uniqueness in their mechanical response [43]. Also known as bifurcation, loss of uniqueness is a point in the strain-stress path in which several displacement increments become possible for a unique stress increment. Bifurcation poses several problems in numerical implementations: in first place it degrades performance of zero-finding algorithms e.g., Newton method; those can jump from the neighbourhood of one possible solution to another without ever converging to a solution. In second place, strain localization $[44,45]$ resulting from the lack of a characteristic length in the classical definition of the Cauchy stress $[46,47]$ causes mesh dependency. Numerical methods have been developed to overcome the bad performance of zero-finding algorithms [48] and regularization techniques both in nonlocal [49] and local forms [50,51] address the ill-posedness of the Cauchy expression and mesh dependency. Second gradient model, as a particular case of the Germain theory [51] is one of the available local regularization techniques [52-58]. Second gradient has been integrated in numerical codes and extensively applied in geomechanics and geotechnics applications with satisfactory results [59-62]. Despite improvements in zero-finding algorithms and regularization techniques, numerical models both in the micro- and macro-scales can still present bifurcation and instability $[63,64]$.

Because of measurement uncertainties, loss of uniqueness translates into non-determinism in real materials. Numerical models, which are deterministic by default, need to account for non-determinism to properly reproduce natural phenomena. This is usually accomplished by introducing artificial material heterogeneity. Several works study the effects of material heterogeneity and its relation with strain localization [65-67]. The introduction of material defects and material heterogeneity in the problem description or numerical discretization are common in geomechanics [68-70], these approaches remain very pragmatic and do not question the nature of the material heterogeneity. Indeed, the micro-macro issue of localization onset and its relation with inhomogeneity are still under intense research [71-74]. General results about possible links between material stability and global stability are still missing [43]. The study of intrinsic material non-determinism as source of heterogeneity is the very subject of the present work. The main novelty is the use of a data-driven ML approach instead of a parametric random field for the reproduction of material heterogeneity.

In this paper, a micro-structure issued from the asymptotic homogenization of a poroelastic matrix with damageable cracks is used to generate a synthetic database of failure states. Normally, this database could be used to build a failure surface delimiting the non-failed space. Unicity and determinism of the solution are evaluated and a series of ML algorithms used to properly reproduce the micro-scale response. The paper is organized as follows: in Section 2 the micro-scale, damage criterion and non-unicity problems are formulated, in Section 3 the different ML algorithms and training results presented, in Section 4 the result from a trained generator is used to provide material constitutive responses. Paper ends with discussion in Section 5 and conclusions in Section 6. 


\section{Problem Description}

\subsection{Micro-Scale Problem}

The strong formulation of the micro-scale equations was obtained using asymptotic homogenization [75] in the framework of small strains using multiscale asymptotic expansions [76], full description of the expansions is detailed in Argilaga et al. [25]. The original problem being hydromechanical, the hydraulic and mechanical parts can be uncoupled and only the mechanical part is considered in the present application. A 2D micro-scale structure consisting of a porous elastic matrix and a 1D thin soft layer [77] constituting a crack network is considered. Crack elastic properties are linear in the normal and tangential components and both compression and extension, cracks undergo damage subject to opening. The elementary geometry is periodic in both $x$ and $y$ dimensions. This micro-scale is representative of materials such as coal, crystalline rocks or composites.

The strong formulation of the problem (mechanical) reads:

$$
\begin{gathered}
\operatorname{div}^{y} \sigma^{(0)}=0, \text { in } Y \\
\sigma^{(0)} \cdot \vec{n}=\vec{T}^{(0)}, \text { on } \Gamma^{Y} \\
\sigma^{(0)}=c:\left(\epsilon^{x}\left(\vec{u}^{(0)}\right)+\epsilon^{y}\left(\vec{u}^{(1)}\right)\right)-p^{(0)} \alpha \\
\vec{T}^{(0)}=G \cdot\left[\left[\vec{u}^{(1)}\right]\right]-p^{(0)} \vec{A}, \text { on } \Gamma^{Y} \\
\vec{u}^{(1)}, \sigma^{(0)} Y \text {-periodic, }
\end{gathered}
$$

where $Y$ is the elementary periodic cell and $\Gamma^{Y}$ its boundary, $\vec{u}$ the displacement field, in the macro $\vec{u}^{(0)}$ and micro $\vec{u}^{(1)}$ scales, $\sigma^{(0)}$ is the total Cauchy macro stress tensor, $p^{(0)}$ the macro pore pressure, $c$ the fourth order tensor of elastic stiffness and $\alpha$ the second order tensor of Biot coefficients. Similarly to Equation (3), Equation (4) is the Biot equation for the crack network, $G$ and $\vec{A}$ are the elastic coefficients of the cracks and its Biot tensor. The stress vector $\vec{T}=\sigma \cdot \vec{n}$ is continuous on the crack jumps and the displacement field $\vec{u}$ discontinuous; discontinuity is the micro displacement field is denoted by $\left[\left[\vec{u}^{(1)}\right]\right]$.

\subsection{Crack Network Damage}

It is considered that damage is concentrated solely in the crack network, this assumption holds true while damage depends on strain and the crack network is significantly softer than the porous matrix. Equation (4) becomes:

$$
\vec{T}^{(e)}=\left(1-d^{(0)}(\tau)\right) G \cdot\left[\left[\vec{u}^{(1)}(\tau)\right]\right]-p^{(0)}(\tau) \vec{A},
$$

with:

$$
d^{(0)}(\tau)=\sup _{0 \leq \rho \leq \tau} f\left(\frac{\left\|\left[\left[\vec{u}^{(1)}(\rho)\right]\right]\right\|}{\Delta_{n}}\right)
$$

where $f$ is the damage function proposed by Dascalu et al. [78]:

$$
z \stackrel{f}{\rightarrow} f(z)= \begin{cases}z(2-z) & 0 \leq z<1 \\ 1 & 1 \leq z\end{cases}
$$

where $\vec{T}^{(e)}$ is the stress in the damaged crack resulting from the asymptotic expansions $(e), \tau$ the time-history variable of damage $d^{(0)}(\tau), \rho$ the time-history variable of the displacement field $\vec{u}^{(1)}(\rho)$ in the cracks, $\Delta_{n}$ the length feature of the crack damage. 


\subsection{Numerical Model}

The described micro-scale problem is solved using an in-house Finite Element Model. The elementary periodic cell consists of 144 2D 4-node quadrangular elements modelling two types of matrix grains (Figure 1a), node numbering available in the supplementary data, 52 crack nodes modelling the crack jumps (Figure 1b), and 40 linear 2-node elements modelling the crack network (Figure 1c), resulting in a total of 215 nodes. Cracks are initially closed, figure shows cracks with opening for convenience of representation.
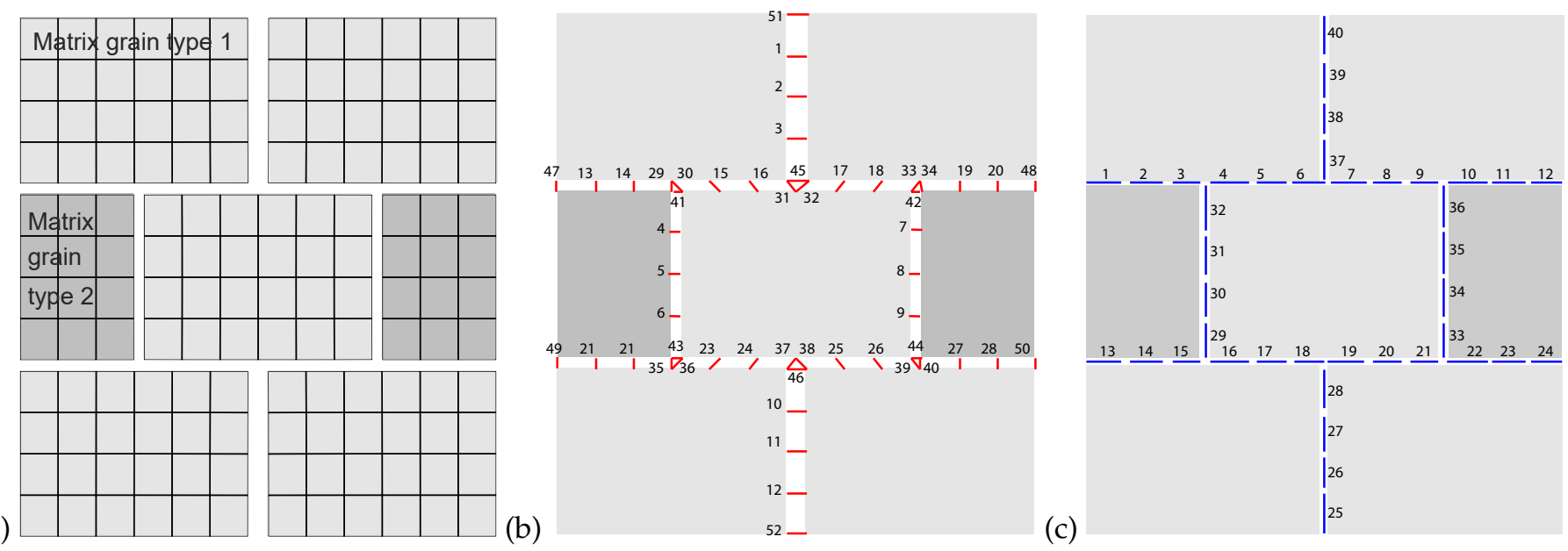

Figure 1. Geometry of the micro-scale Finite Element mesh with two matrix grain types (a), crack network nodes or links (b) and crack network elements containing two Gauss points each (c).

Periodic boundary conditions are enforced by penalization, thus increasing the nonzero count in the global stiffness matrix as well as the bandwidth (Figure 2), which in turn increases the computational load of the solver. In order to minimize eigenvector disparity which can cause poor solver performance, penalization stiffness is limited at $10^{3}$ times the stiffness of the undamaged cracks.
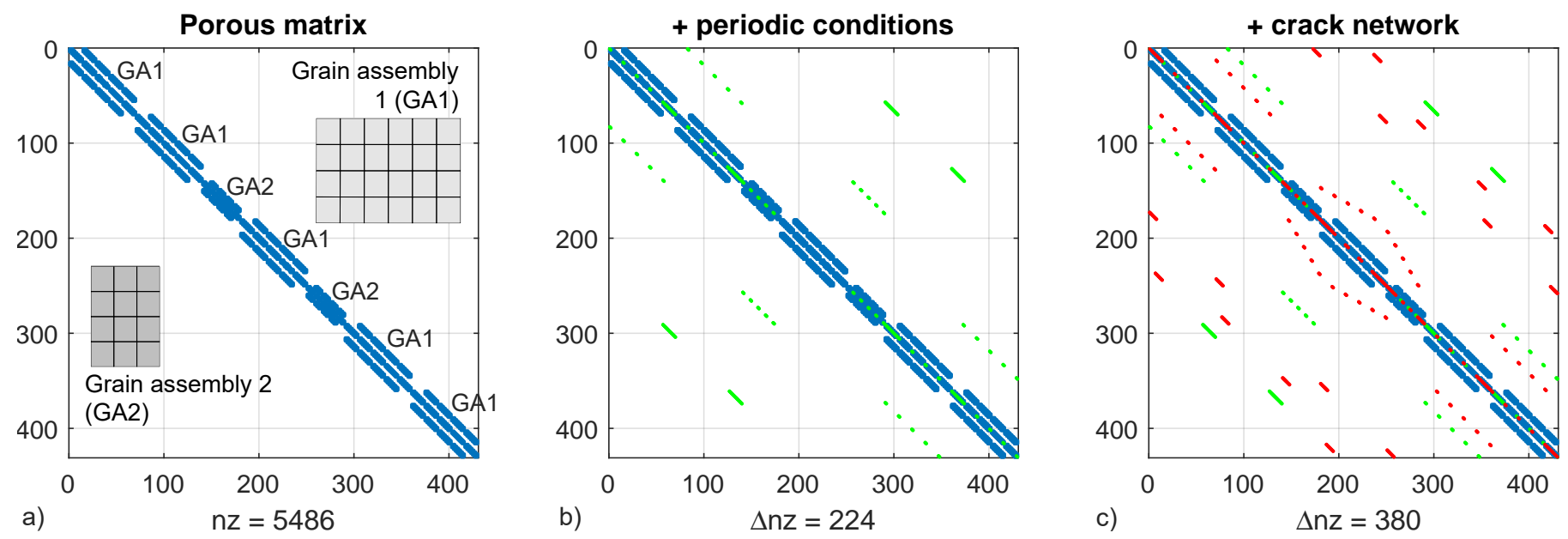

Figure 2. Assembly of the matrix grains in the global stiffness matrix, five grain type 1 assemblies (GA1) and two grain type 2 assemblies (GA2) (a). Assembly of periodic boundary conditions and kinematic restrictions by penalization causing a large increase of matrix bandwidth (b). Crack network assembly (c). Where $n z$ is the non-zero count and $\Delta n z$ the increase of non-zero count by periodic conditions and crack links. 
Matrix elastic properties are defined by the Lame constants: $\lambda=1.442 \mathrm{GPa}$ and $\mu=0.961 \mathrm{GPa}$ (Young's modulus $E=2.5 \mathrm{GPa}$ and Poisson's ratio $v=0.3$ ). These elastic coefficients are borrowed from Marinelli et al. [79] for validation purposes. The problem being non-linear and time-dependent, it is incrementally loaded and secant method is used at each step to find a solution. The loading is macroscopically strain controlled, defined in the space of $\epsilon^{x}\left(\vec{u}^{(0)}\right)$, and the response is macroscopic stress in the space of $\sigma^{(0)}$.

Micro-scale deformation for a loading in each degree of freedom (DoF) is presented (Figure 3), greyscale indicates von Mises stress in Pa. In this case no damage is considered (elastic case), equivalent elastic coefficients of the micro-structure can be obtained via numerical homogenization. The fourth DoF which corresponds to the macro pore pressure has an influence on the micro-structure through the crack network Biot coefficient $\vec{A}$. This influence has proved to be relatively small (see [25], Figure 5), therefore in the following only the first three DoF are considered.

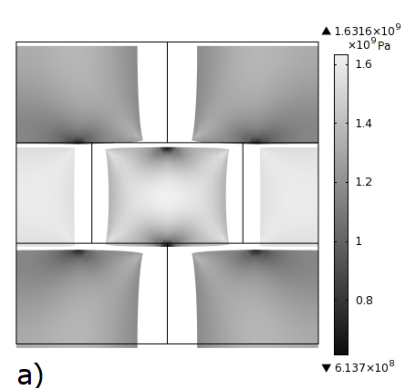

a)

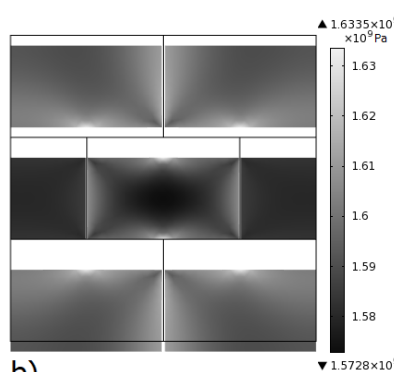

b)

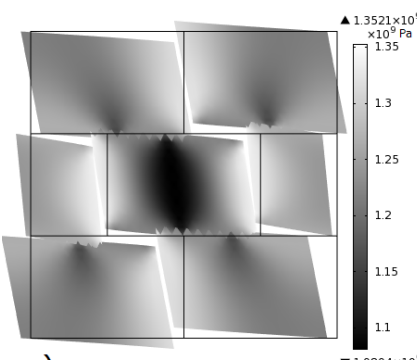

c)

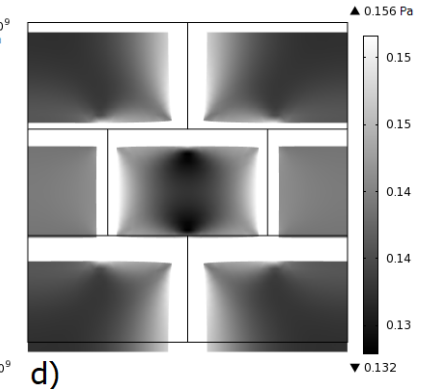

d)

Figure 3. Configurations for macro deformations $\epsilon^{x}=\left[\begin{array}{lll}1 & 0 & 0\end{array}\right](\mathbf{a}), \epsilon^{x}=\left[\begin{array}{lll}0 & 1 & 0\end{array}\right](\mathbf{b}), \epsilon^{x}=\left[\begin{array}{lll}0 & 0 & 1\end{array}\right]$ (c), and $\epsilon^{x}=\left[\begin{array}{ll}0 & 0\end{array}\right], p^{(0)}=1$ (d). Colormap represents von Mises stress in Pascals. Crack stiffness: $\mathrm{G}=1 \times 10^{13} \mathrm{~Pa}$. Deformation magnification: $10 \times$.

\subsection{Failure Criterion}

A failure criterion is defined accounting for the concept of structural integrity of the overlying mechanical problem [2]. The criterion is as follows: if the force magnitude defined in Equation (9) decreases a given quantity from a peak, the sample is considered to be failed in the context of the overlying mechanical problem; Equation (10):

$$
\begin{gathered}
f m_{c}=\left\|\sigma_{i j}\right\| \\
f m_{c}<r_{f m}\left(\sup _{0 \leq \rho \leq \tau}\left(f m_{c}(\tau)\right)\right),
\end{gathered}
$$

where $r_{f m}$ is the peak force ratio. A peak ratio is used because a material point failure does not automatically cause loss of controllability in the overlying mechanical problem [80]. Due to the stiffness of the neighbouring material, the structure can accommodate the new stress distribution without further propagating material damage. A value $r_{f m}=0.8$ is selected as a compromise between high values which fail when reaching the stress peak and low values which create unbreakable structures. Examples of unbreakable micro-structures: https:/ / doi.org/10.6084/m9.figshare.14540118.v1 (posted on 5 May 2021).

The input feature of the model is the loading vector $\epsilon^{x}\left(\vec{u}^{(0)}\right)$ and the output feature, which is defined in the same space as the input, is the strain magnitude corresponding to the micro-structural failure. All loadings start at an unstrained configuration i.e., $\epsilon^{x}\left(\vec{u}^{(0)}\right)=(0,0,0)$ and evolve in a linear manner. A sampling of the loading space, either random or systematic allows to obtain the input and output datasets to be used later in the ML algorithm. Assuming determinism of the constitutive response (classical hypothesis), a finite number of failure points can be used to define a boundary between the anelastic region and the failed region in the strain space. This boundary is usually known as failure 
envelope and parametrized in a variety of failure theories e.g., Mohr-Coulomb, DruckerPrager or Van Eckelen. A failure envelope of the proposed micro-scale model is generated by applying a succession of equidistant loadings in the space $\epsilon^{x}\left(\vec{u}^{(0)}\right)=\left(\epsilon_{x x}, \epsilon_{y y}, \epsilon_{x y}\right)$, in this example the shear component $\epsilon_{x y}$ is taken equal to zero for sake of visualization. Due to the symmetries of the model the response presents a revolution symmetry which allows to reduce the computational load to one half by only sampling the positive values of $\epsilon_{y y}$. One sample is defined as the input/output pair obtained from a single loading path (Figure $4 \mathrm{a}$ ). The resolution of the sampling is defined as the number of samples in the loading angle interval $[0, \pi)$ and in the present example is 64 (Figure $4 \mathrm{~b}$ ).

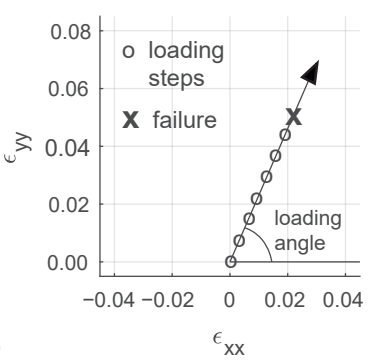

(a)

Figure 4. Strain space macroscopic incremental loading (a). Failure surface in the $x x-y y$ strain space separating the anelastic non-failed region and failed region, 64 samples resolution (b).

The 64 sample resolution surface envelope presents a jump in four regions of the $x-y$ strain space. This results in a non-convex surface which is one of the reasons why meta-heuristics have been used to optimize the problem in previous works [2].

\subsection{Loss of Uniqueness}

Uniqueness can not be assured in problems presenting material softening e.g., [43]. It is proved that when stiffness decreases under loading, the discharge branch can have any slope; and the solution cannot be determined a priori, thus the problem becoming non-deterministic. In a PDE formulation, when any of the eigenvalues of the stiffness tensor becomes zero, the problem loses its ellipticity and any strain applied in the direction of the vanishing eigenvalue fulfils the equilibrium condition, thus making the solution non-unique. Loss of uniqueness, or bifurcation, in numerical models is an interesting phenomenon that agrees with experimental observations; nevertheless, it represents a numerical challenge i.e., decreased efficiency of Newton method, no warranty of finding all possible solutions, main concern in e.g., reliability or failure analysis.

In the following some of the solutions for a biaxial compression test of the presented micro-scale are explored. This is not an exhaustive search to find all solutions but a demonstration of the existence of more than one solution. The asymptotic result of the homogenized stiffness $\sigma_{2222}^{H}$ for a strain $\epsilon_{11}=0.85 \%$ is obtained in a parametric study on the number of time steps and tolerance threshold of the Newton algorithm, 2400 computations are performed (Figure 5a). Both vertical and horizontal axes represent discrete values of tolerance and number of steps respectively, thus the plot is not a stress field but rather the representation of individual stress samples. These results showcase the non-uniqueness of the model; infinitesimal variations of the input cause finite variations of the output. Stress values are polarized in two regions, one around $\sigma_{2222}^{H}=2.1 \times 10^{8} \mathrm{~Pa}$ and another around $\sigma_{2222}^{H}=2.6 \times 10^{8} \mathrm{~Pa}$, (Figure $5 \mathrm{~b}$ ). Two loading histories are presented to showcase the first and second solution attractors (Figure $5 \mathrm{c}$ ). 

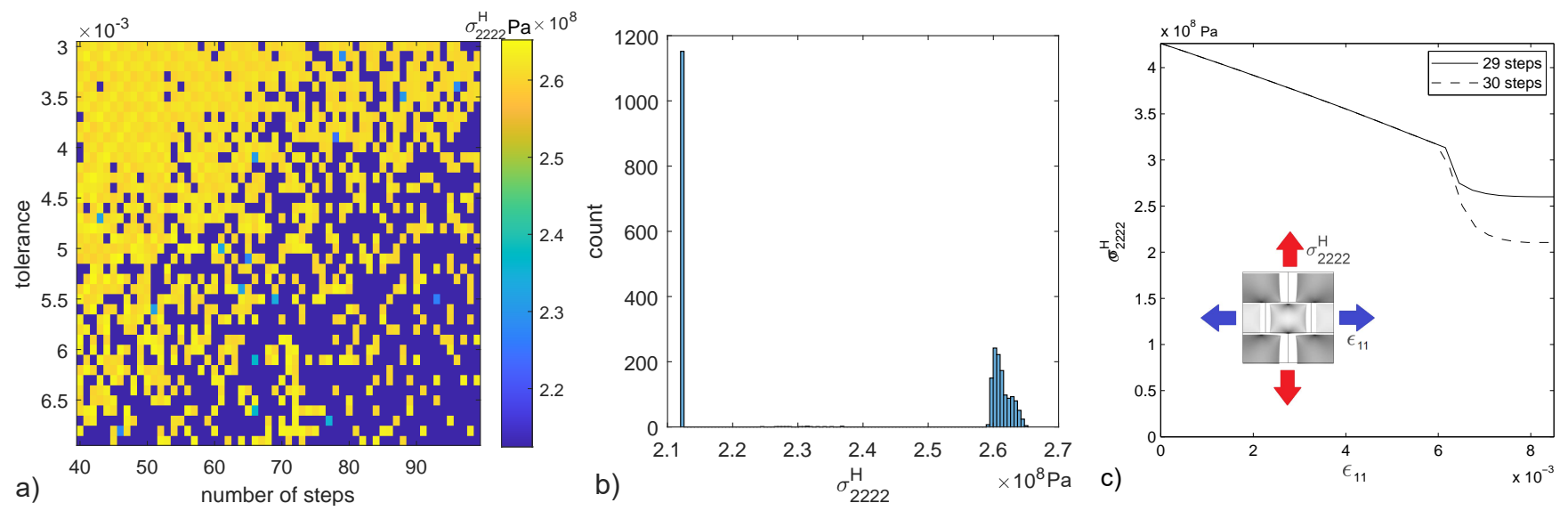

Figure 5. $\sigma_{2222}^{H}$ value for the 2400 cases of the parametric test (a), histogram of the $2400 \sigma_{2222}^{H}$ values (b) and stress-strain history for two cases representative of the two main groups of solutions seen in the histogram (c).

Parametric test results (Figure 5a) present a pattern of $\sigma_{2222}^{H}$ which seems to follow a random distribution, with the top left part presenting a higher probability of values close to $\sigma_{2222}^{H}=2.6 \times 10^{8} \mathrm{~Pa}$ and the bottom right region close to $\sigma_{2222}^{H}=2.1 \times 10^{8} \mathrm{~Pa}$. In addition, $\sigma_{2222}^{H}$ histogram (Figure $5 b$ ) shows that low values seem to concentrate in one narrow region while high values present a distribution in the range $\sigma_{2222}^{H}=[2.60-2.65] \times 10^{8} \mathrm{~Pa}$.

Moire Fringe interference plots (Figure 6) discover some patterns in the previous $\sigma_{2222}^{H}=[2.60-2.65] \times 10^{8}$ Pa stress results in the top left region of (Figure 6a) as well as in a new parametric study with more stringent tolerance values and more samples (Figure 6b), in the latter the pattern appears to depend only on number of steps, not on tolerance threshold. The differences between cases 1 and 2 (Figure 6) indicate that the observed patters are of numerical precision nature and can be predicted by adjusting numerical parameters. Despite the existence of predictable patterns in specific conditions, stress results (Figure 5a) are a demonstration of the non-determinism of the present numerical model. Next section explores micro-scale failure accounting for its non-deterministic nature.
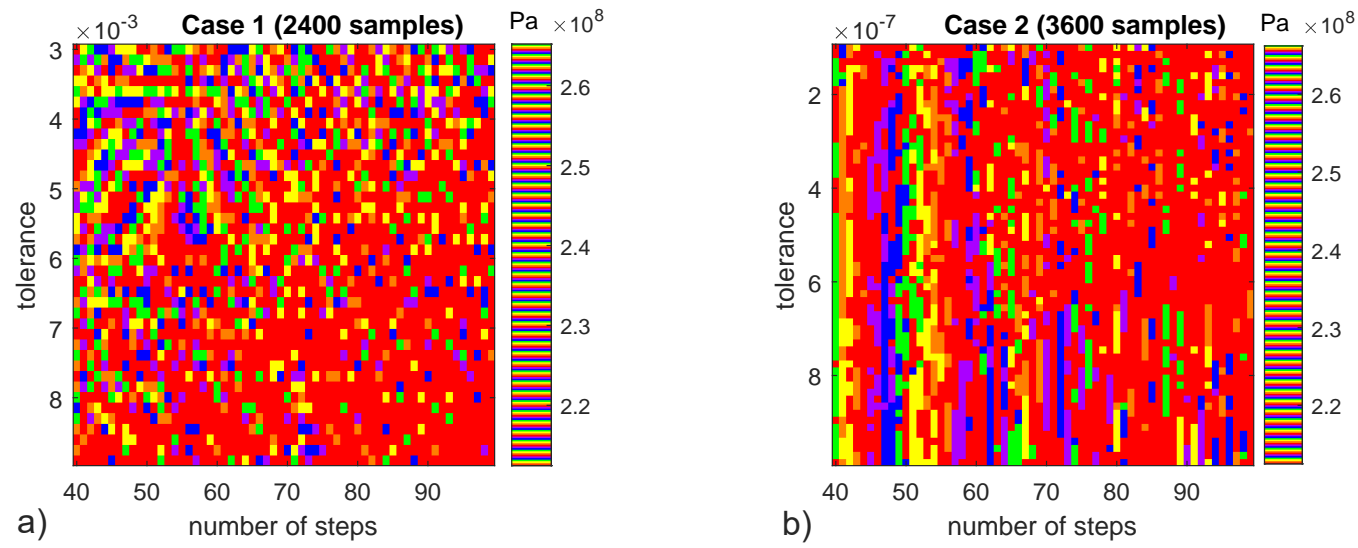

Figure 6. Moire Fringe interference patterns for two cases of number of steps and Newton method tolerance. For some regions of $\sigma_{2222}^{H}$ results seem to follow a predictable pattern while in others there is only random noise. Colormap "prism" in Matalb is used to obtain the interference. (a) 2400 sample. (b) 3600 sample.

\subsection{Failure Sampling}

A reference micro-scale configuration is used to study the non-unique response according to the criteria defined in Equation (10). Similarly to the previous Moire Fringe interference study, the number of loading steps and tolerance threshold are used as numerical variability to trigger different solutions. A range between 195 and 205 loading steps $(n)$ 
is adopted, its value is randomly determined at each simulation with an equal probability for all the range. Tolerance (tol) range is set to follow a uniform distribution between 0.001 and 0.0001. Parameters of the different micro-scale configurations can be found in Table 1 and samples are drawn using a uniform random distribution of the loading angle.

Table 1. Micro-scale parameters used in the different parametric studies.

\begin{tabular}{cccccccc}
\hline Configuration & $\boldsymbol{\Delta}_{\boldsymbol{n}}$ & $\boldsymbol{\mu}\left[\mathbf{1 0}^{\mathbf{6}} \mathbf{P a}\right]$ & $\boldsymbol{\lambda}\left[\mathbf{1 0}^{\mathbf{6}} \mathbf{P a}\right]$ & $\boldsymbol{G}\left[\mathbf{1 0}^{\mathbf{1 3}} \mathbf{P a}\right]$ & $\mathbf{T o l}$. & $\mathbf{n}$ & $f_{m c}$ \\
\hline$\mu$-scale 1 & 0.0050 & 961 & 1442 & $6 \times 10^{13}$ & {$[0.001-0.0001]$} & {$[195-205]$} & 0.9 \\
$\mu$-scale 2 & 0.0025 & 961 & 1442 & $6 \times 10^{13}$ & {$[0.001-0.0001]$} & {$[195-205]$} & 0.9 \\
$\mu$-scale 3 & 0.0025 & {$[480-1442]$} & {$[721-2163]$} & $6 \times 10^{13}$ & 0.0001 & 200 & 0.9 \\
$\mu$-scale 4 & 0.0050 & 961 & 1442 & $1 \times 10^{13}$ & 0.0001 & 200 & 0.8 \\
$\mu$-scale 5 & 0.0050 & 961 & 1442 & $6.5 \times 10^{13}$ & 0.0001 & 200 & 0.8 \\
$\mu$-scale 6 & 0.0050 & 961 & 1442 & $6.0 \times 10^{13}$ & 0.0001 & 200 & 0.8 \\
\hline
\end{tabular}

The micro-scale 1 has a standard set of parameters similar to the one used in Argilaga and Papachristos [2], in this case with a stiffer crack network. Micro-scale 2 decreases $\Delta_{n}$ to one half with respect to micro-scale 1 . Results of cases 1 and 2, with 400 samples each, present failure points that clearly define a failure surface, a group of points in the vicinity of the surface but scattered in a diffuse region, and some points at a further distance (Figure 7, $a$ and $b$ ), the second case (micro-scale 2) presents lower strain values due to the decrease in $\Delta_{n}$. (Figure 7c) shows a detail of the micro-scale 2 in this case with 5000 samples.
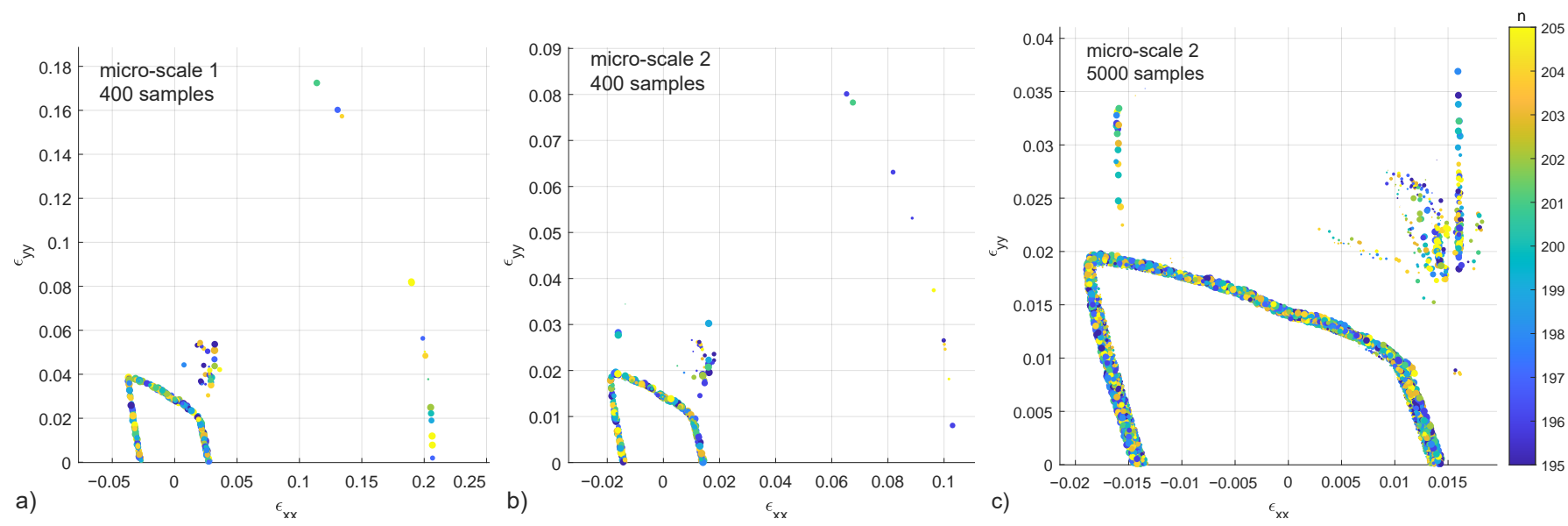

Figure 7. Failure points in the $x x$-yy strain space for the micro-scale 1, 400 samples (a), micro-scale 2, 400 samples (b), detail of micro-scale 2, 5000 samples (c). Colorbar represents the number of steps [195-205] and the diameter of the dots the tolerance threshold [0.001-0.0001]. Files available in the supplementary materials.

Micro-scale 3 (Figure 8) presents a parametric study on physical parameters instead of numerical ones (Table 1 , third row). $\mu$ coefficient follows a uniform distribution in the range [480-1442] $\times 10^{6} \mathrm{~Pa}$ and $\lambda$ in the range [721-2163] $\times 10^{6} \mathrm{~Pa}$. In this case, the scattering due to the non-unique response of the constitutive law is combined with the physical influence of $\mu$. There is a higher number of samples with lower values of $\mu$ further away from the origin, $\epsilon^{x}=(0,0,0)$, this is due to reaching the state of "unbreakable structure" mentioned before, also lower values of $\lambda$ show higher strain at failure. 


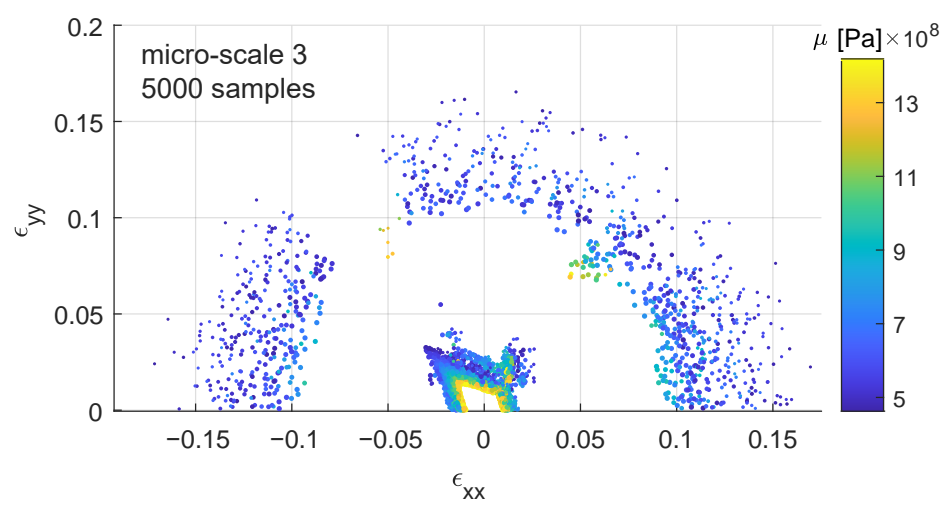

Figure 8. Failure points in the $x x-y y$ strain space. Colorbar represents $\mu$ coefficient in the range [480-1442] $\times 10^{6} \mathrm{~Pa}$ and the diameter of the dots the tolerance threshold [0.0010-0.0001]. File available in the supplementary materials.

Micro-scales 4 and 5 (Table 1) are not fed with the variation of any parameter, but only with random uniform sampling of the loading angle both in the $x x-y y$ and $x x-x y$ planes. Shear strain $\epsilon_{x y}$ DoF is added in order to obtain solutions in the full loading space (Figure 9). The difference between (Figure 9) a and $b$ is the crack stiffness $G$ being 6.5 time larger in the latter, causing many of the samples to represent an "unbreakable structure".
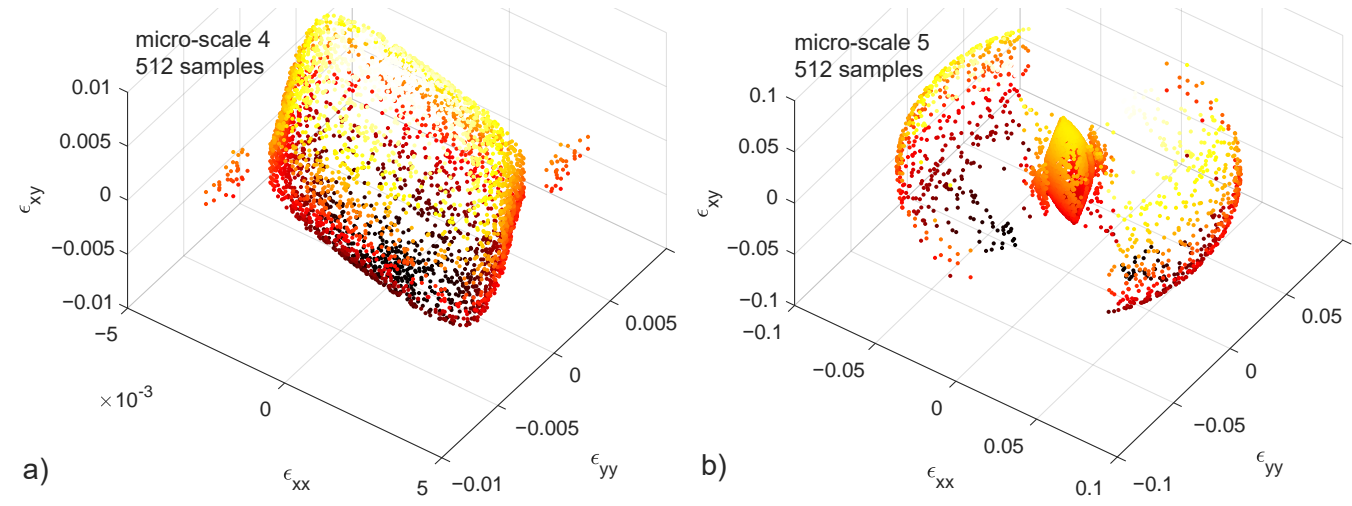

Figure 9. Failure points in the $x x-y y-x y$ strain space. 512 samples in each case. Colorbar represents the value of $\epsilon_{x y}$. Crack stiffness $G=1 \times 10^{13}(\mathbf{a})$ and $G=6.5 \times 10^{13}(\mathbf{b})$. 3D figures available in the supplementary materials.

The last configuration is retained because it generates samples in the three known test fates i.e., failure in the actual failure surface, diffuse samples in the neighbourhood of the surface and points far from the origin due to an "unbreakable structure" phenomenon. A slight modification leads to the creation of the micro-scale 6 (Table 1) by reducing the crack stiffness $G$ to $6.0 \times 10^{13}$. This is done to decrease the weight of samples with "unbreakable structure" state.

\section{Failure Modelling}

In this section, several ML algorithms are evaluated for the failure modelling of the micro-scale. In order to find the least complex ML algorithm capable of reproducing the micro-scale response, three representative ML candidates with increasing levels of supervision and network depths are selected. Given the characteristics of the micro-scale response, ML candidates are selected to be either good at non-deterministic reproduction, clustering, or both of them. 


\subsection{Gaussian Process Regression}

A Gaussian Process Regression (GPR) is a supervised learning method with great flexibility to adapt to unknown data distributions. GPR is a non-parametric kernel-based stochastic model [81], differently from simpler methods such as least-squares, in GPR it is not needed to relate the approximated function to a specific model. This feature makes it suitable for the present application in which the data distribution is a priori unknown. In addition, GPR gives a measure of uncertainty for the predictions which can reproduce the non-determinism of the present application. A GPR model is trained using 1024 samples of the micro-scale 6 in the strain plane $\epsilon_{x y}=0$. The training dataset is kept small because computational time increases cubically with its size due to the required matrix inversion. Predictor data is generated by calculating the loading angle $\gamma$, Equation (11):

$$
\gamma=\operatorname{atan}\left(\frac{\epsilon_{y y}}{\epsilon_{x x}}\right)
$$

and the true variable is the norm of the strain vector at failure:

$$
\|\epsilon\|=\left(\epsilon_{x x}^{2}+\epsilon_{y y}^{2}\right)^{1 / 2} \text {. }
$$

A hyperparameter optimization is performed using a constant basis function, kernel functions are: Rational Quadratic, Squared Exponential, Matern 5/2, Matern 3/2 and Exponential. The best fit is obtained with the isotropic exponential kernel function (Figure 10).

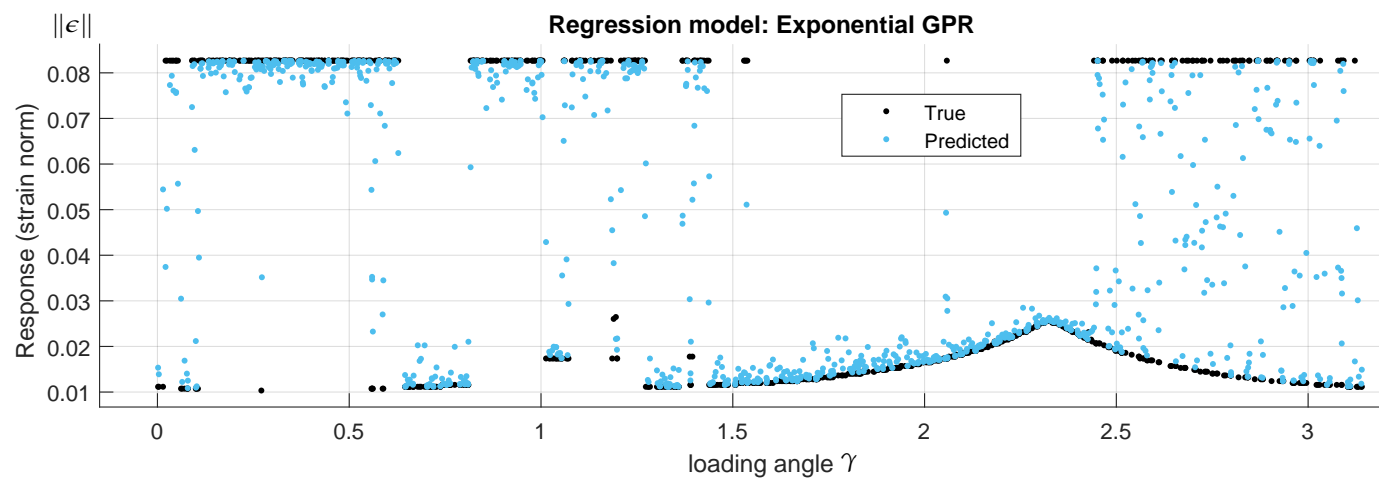

Figure 10. True and predicted response using an Exponential Gaussian Process Regression (GPR) deep learning algorithm.

With fitting metrics: $R M S E=0.015452, R^{2}=0.79, M S E=0.00023876$ and $M A E=0.0064823$. Despite the relatively good metrics, predictions show many points falling in between the main attractors in real data (Figure 10). In GPR, a Bayesian approach infers a probability distribution over all the data; in the present case, and due to the presence of different failure modes and therefore different distributions, GPR fails at successfully modelling the response. A method capable of identifying the different failure modes is needed in either a stand-alone application or in conjunction with GPR.

\subsection{Neural Net Clustering}

In this subsection neural networks are used to cluster data belonging to the different failure modes. In clustering problems data is grouped according to the similarity of given characteristics. This allows to build a Self-Organizing Map (SOM) which is a low dimensional representation (typically 2D) of a higher dimensional data while conserving its topology [82]. Networks are trained with unsupervised weight and bias learning rules with batch updates i.e., weights and biases are updated at the end of an entire pass through the input data. Network topology consists of one input, one output and one hidden layer 
with 256 neurons (Figure 11). The input size is 2 corresponding to the strain components $\epsilon_{x x}$ and $\epsilon_{y y}$. A training is performed using 12,000 samples of micro-scale 6.

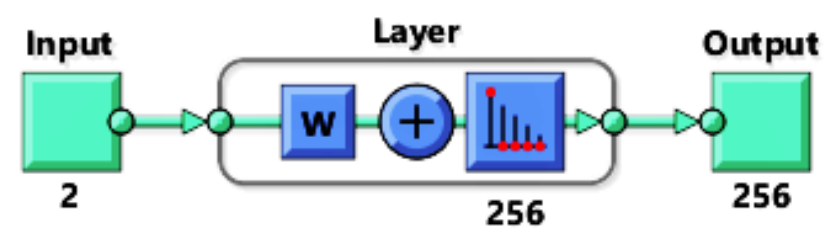

Figure 11. Neural net topology with $16 \times 16$ neurons in the hidden layer.

Net topology in the hidden layer consists in a honeycomb arrangement of $16 \times 16$ hexagonal neuron cells (Figure 12a), each cell has six connections with neighbouring cells (Figure 12b), other organizations are also possible. In this case both SOM representation and data are 2D. SOM weight distances map (Figure 12c) presents low values when weights are tightly clustered (yellow cells) and high values separating weights in different feature groups (red and black cells). From this results three categories can be clearly established.
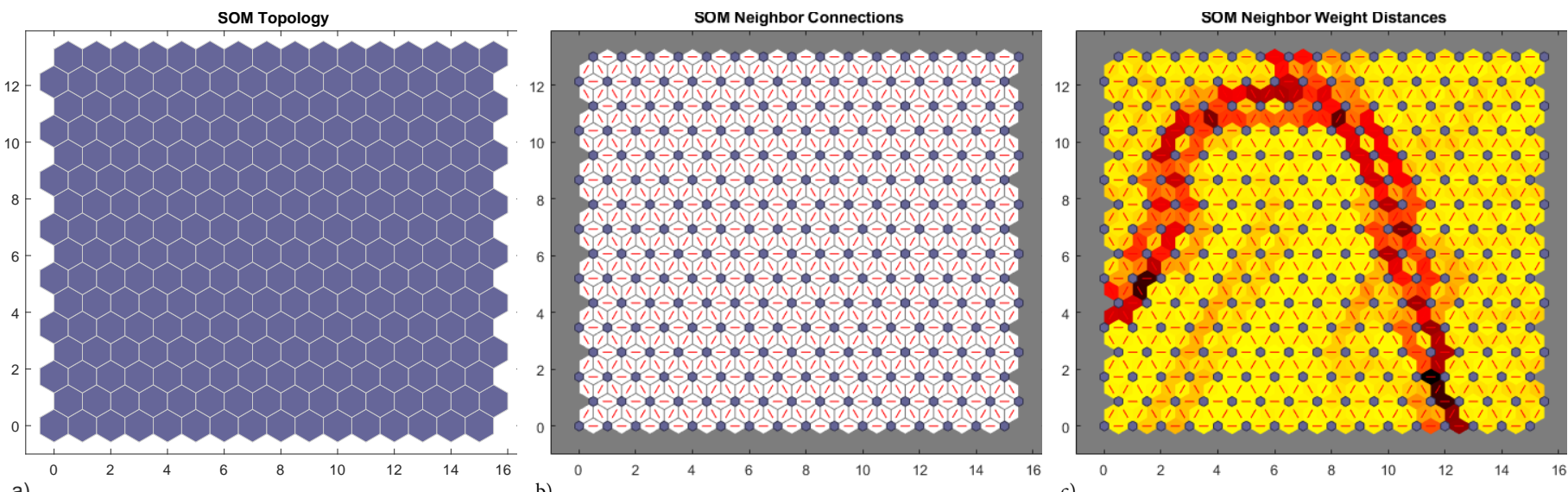

Figure 12. Neural net clustering 12,000 samples, $2 \mathrm{D}$ strain $\epsilon_{x x}-\epsilon_{y y} .16 \times 16$ topology (a), connections (b) and weights (c).

SOM weight positions show the correct identification of the inner surface as a feature cluster (Figure 13a); where green spots are the training vectors, blue spots the neuron's weights vector and red lines are weight connections or distances. The outmost weights (corresponding to unbreakable micro-structures) are erroneously split between two features. Hits count plot (Figure 13b) shows the feature connections per neuron of the previous. Data evenly distributed across neurons is best for a correct clustering; the highest value of connections per neuron is 286 and neurons between features display zero connections, but overall the data is well distributed within features (Figure 13b).

The scattered group of points in the vicinity of the inner failure surface is not clearly identified as pertaining to a unique feature, therefore the SOM with $16 \times 16$ neurons fails at clustering the constitutive response. Other network topologies and training data sizes have been tested with similar results. 

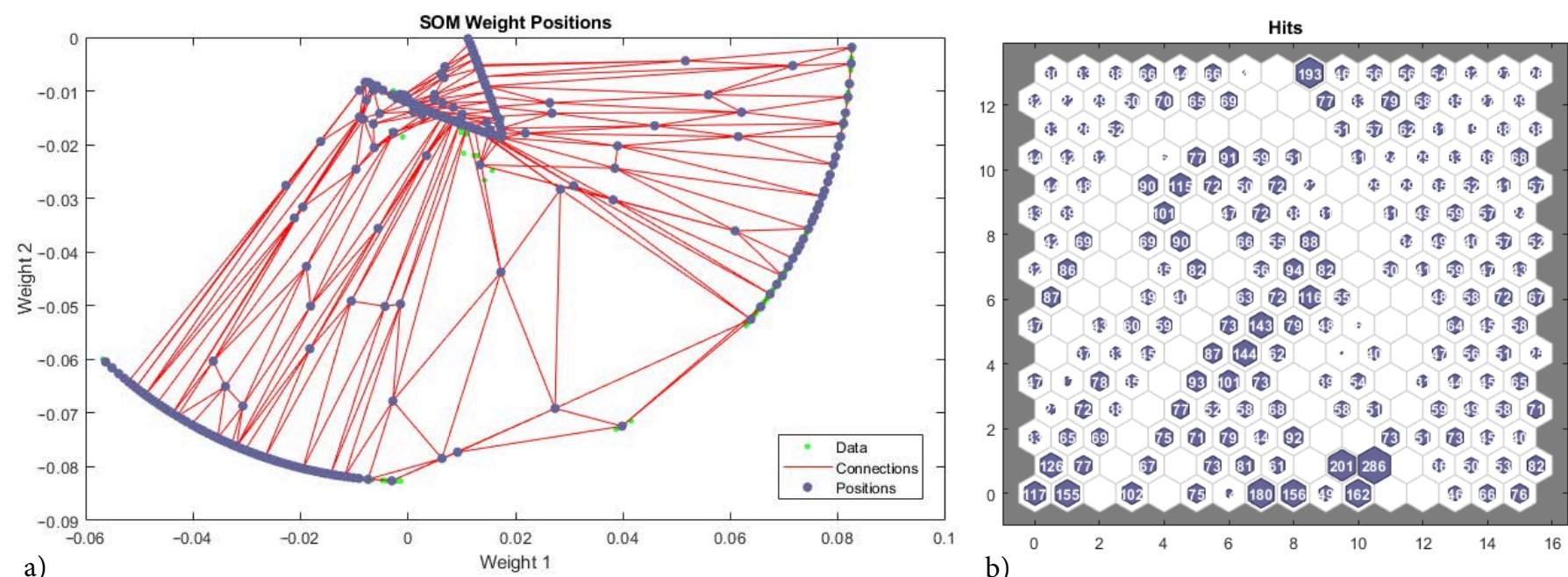

Figure 13. Neural net clustering 12,000 samples, $2 \mathrm{D}$ strain $\epsilon_{x x}-\epsilon_{y y} .16 \times 16$ topology. Weight positions and hits. (a) SOM weight positions. (b) Hits count plot.

\subsection{Generative Adversarial Networks}

Generative Adversarial Networks (GANs) first proposed by Goodfellow [83] is a deep learning approach in which the discriminative model itself is a neural network. Many GANs variants have been proposed since its initial introduction in 2017: Least Squares Generative Adversarial Networks (LSGANs) by Mao et al. [84], Deep Convolutional Generative Adversarial Networks (DCGANs) by Radford et al. [85], Wasserstein Generative Adversarial Networks (WGANs) by Arjovsky et al. [86], among others and they play an important role in the generation of artificial images. A collection of the main GANs codes for Matlab can be found in the repository: https://github.com/zcemycl/Matlab-GAN (accessed on 18 October 2021).

In a GANs a Generator (Gen) consisting in a deep neural network generates samples to imitate the ones of the training distribution while the Discriminator (Dis) gives the probability of the generated samples coming from the generator or from the training dataset. The objective of the training process is for the Gen to maximize the probability of the Dis to make a mistake. At the end of the training the Dis can be dismissed and the Gen fed with random noise $p_{z}(z)$ to generate synthetic samples. This two-sided minmax game can be written using the value function $v(D i s, G e n)$ as:

$$
\min _{\text {Gen }} \max _{\text {Dis }} v(\text { Dis, Gen })=\mathbb{E}_{x \sim p_{\text {data }}(x)}[\log (\operatorname{Dis}(x))]+\mathbb{E}_{Z \sim p_{z}(z)}[\log (1-\operatorname{Dis}(\operatorname{Gen}(z)))],
$$

where $\operatorname{Dis}(x)$ is the probability of $x$ coming from the data rather than the Gen distribution $p_{G e n}$. Dis and Gen are simultaneously trained in order to assign the correct tag to data samples and Gen samples (Dis) and to minimize $\log (1-\operatorname{Dis}(\operatorname{Gen}(z)))($ Gen). GANs schematics (Figure 14) depicts two paths of samples going through the discriminator, it is worth noting that the discriminator does not know which one comes from the real data pool and which one from the Gen, only once the prediction is made the nature of the sample is revealed in order to update Dis and/or Gen with the errors and gradients. 


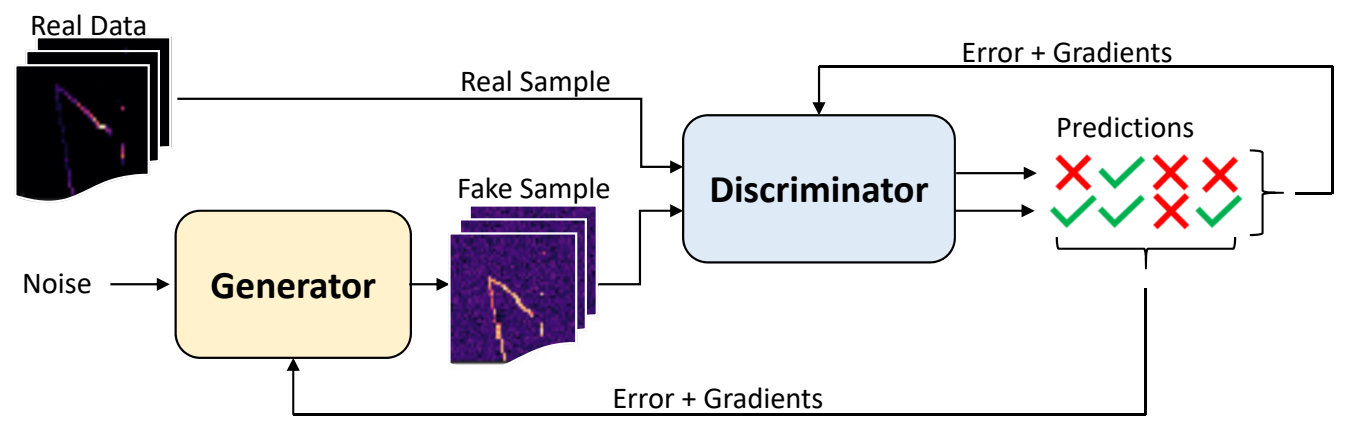

Figure 14. Generative Adversarial Networks (GANs) schematics where the green ticks and red crosses are correct and wrong predictions from the Discriminator respectively.

Here, the original implementation by Goodfellow [83] is used with some modifications in both Gen and Dis architectures. Both Gen and Dis multilayer perceptrons have been modified by reducing their depth, resulting in four layers of neurons in each network. This reduction is done to avoid the "Helvetica scenario" or mode collapse, a known problem in GANs exacerbated by large numbers of layers and neurons [87]. The architecture of both Gen and Dis is presented in Table 2. Latent dimension is a layer of neurons to accommodate the size of the input data to the Gen architecture, then Gen expands through the layers until reaching the image size. In the other hand, the discriminator starts with an input layer equal to the output of the generator and it narrows down to one single neuron in the output layer (binary real or fake prediction).

Table 2. Multilayer perceptron architecture of both Generator and Discriminator.

\begin{tabular}{ccc}
\hline Layer/Neurons & Generator & Discriminator \\
\hline Input Layer & Latent dimension & Image size \\
Hidden Layer 1 & 32 & 64 \\
Hidden Layer 2 & 64 & 32 \\
Output Layer & Image size & 1 \\
\hline
\end{tabular}

Training data consists in 12,000 samples obtained from the micro-scale 6 in the 2D strain space $\left(\epsilon_{x y}=0\right)$. This data is partitioned into 24 segments of 500 samples each and one raster image is obtained from each segment by iteratively converting point coordinates into pixel positions and adding one unit to the corresponding pixel value, finally image values are normalized and can be represented in a linear greyscale. Note that in a GANs the images are converted into a stream of pixel values regardless of rows and columns arrangement, therefore, a string coming from $2 \mathrm{D}$ pixels or $3 \mathrm{D}$ voxels can be fed to the GANs without any modification of the architecture. In the present work 2D images have been selected for sake of presentation. Hyperparameters resulting from the tuning with the 12,000 sample data are presented (Table 3). GANs code and sample database available in the supplementary materials.

Table 3. Hyperparameters tuning.

\begin{tabular}{cc}
\hline Hyperparameter & Value \\
\hline Latent dimension & 64 \\
Batch size & 24 \\
Image size & $128 \times 128$ \\
Dis learning rate & 0.0003 \\
Gen learning rate & 0.0003 \\
Gen decay & 0.201 \\
Gen squared decay & 0.799 \\
Max epochs & 150 \\
\hline
\end{tabular}


The trained Gen can successfully generate images matching the distribution of the 24 ones created with real data. Images are probability maps that, given a strain path, can give the probability of a micro-structure to fail at a given loading level. Assuming a monotonic loading step by step Bayesian conditional probability can also be obtained from the generated probability maps. Real data from micro-scale 6, real probability and generated probability maps are presented (Figure 15).

Real probability sampling pool (Figure $15 b$ ) is a probability map of the entire sampling population of 12,000 points or the 24 raster images stacked and normalized in a single one. While the generated probability map (Figure 15c) is one realization of a trained Gen, thus the first does not directly correlate to the latter. GANs results show the ability to reproduce the given sample distribution including the inner failure surface, diffuse failure region and end of loading without failure. The GANs has been properly tuned to avoid mode collapse in the diffuse failure region and several high probability points can be observed (Figure 15c).
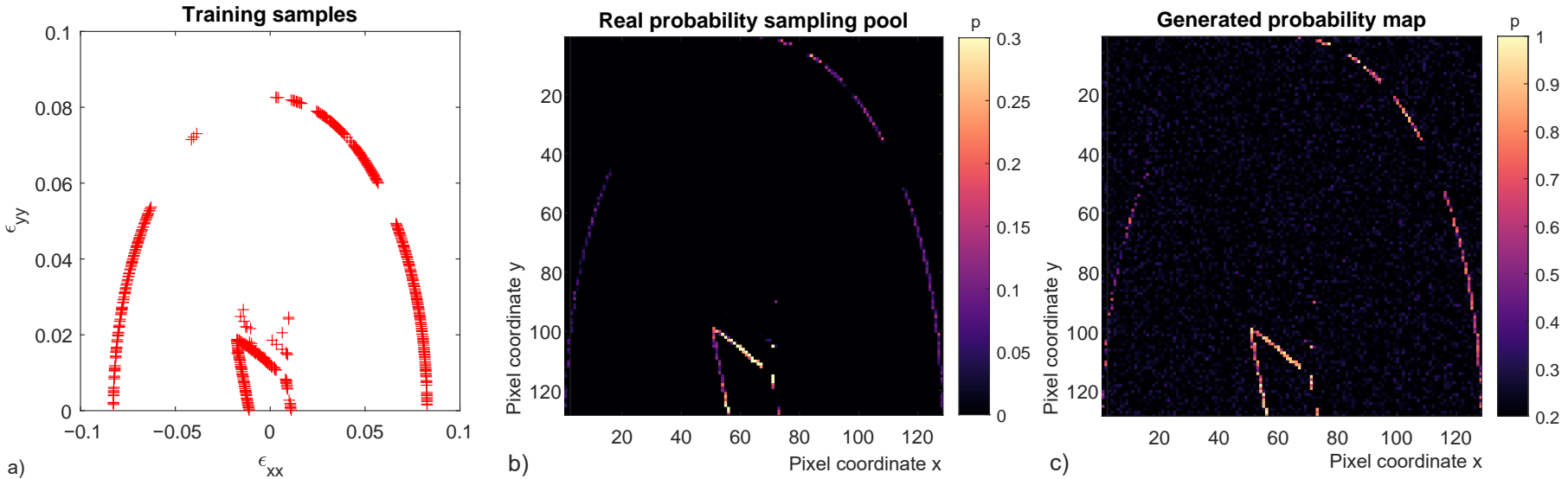

Figure 15. Real data from micro-scale 6 (a), real probability sampling pool (b) and one generated probability map with GANs (c). The three micro-structure fates can be identified in each of the three figures i.e., inner failure surface, diffuse failure region and end of loading without failure.

\section{Application to a Material Point Loading}

GANs is retained as the best performing out of the three tested algorithms. A GANs generated probability map $I$ is used to obtain failure probabilities for given loading paths $\vec{\epsilon}$. A step by step loading is adopted as in the original micro-scale model presented in Section 2. The step size is chosen such as the end of loading is predicted at step 200, this initial estimation is made assuming a homogeneous micro-strain field. The GANs generated image $I$ has a noise floor which needs to be filtered to properly identify failure probabilities. A threshold 50\% above the RMS is adopted to filter the unwanted image noise. Results for three $\vec{\epsilon}$ slopes are presented (Figure 16), where $\tau$ is the history of strain increments.

To refer to the different failure mechanisms, those will be labelled as 1, 2 and 3 from the lower to the higher strain magnitudes. Therefore, failure mechanism 1 is represented by the innermost failure surface, failure mechanism 2 is the diffuse space of scattered failure points, and failure mechanism 3 is not a material failure but the end of loading adopted in the numerical micro-scale model.

Loading case $\epsilon_{22} / \epsilon_{11}=2.0$ (Figure 16, first row) presents a probability density close to $f_{\text {fail }}(\tau)=20 \%$ in loading steps $20-23$ and $f_{\text {fail }}(\tau)=10 \%$ in loading steps 115 and 118 . The accumulated probability density $F_{\text {fail }}(\tau)$ is $82 \%$ past the failure region 1 and $100 \%$ past the failure region 2. Loading case $\epsilon_{22} / \epsilon_{11}=3.7$ (Figure 16, second row) presents a probability density close to $f_{\text {fail }}(\tau)=37 \%$ in loading steps $40-41$ and $f_{\text {fail }}(\tau)=13 \%$ in loading steps 97-98. The accumulated probability density $F_{\text {fail }}(\tau)$ is $73 \%$ past the failure region 1 and $100 \%$ past the failure region 2. Loading case $\epsilon_{22} / \epsilon_{11}=4.1$ (Figure 16, third row) presents a probability density close to $f_{\text {fail }}(\tau)=24 \%$ in loading steps $40-41$ and $f_{\text {fail }}(\tau)=15-20 \%$ in 
loading steps 200-202. The accumulated probability density $F_{\text {fail }}(\tau)$ is $48 \%$ past the failure region 1 and failure region 2 . Probability of reaching end of loading is $52 \%$.
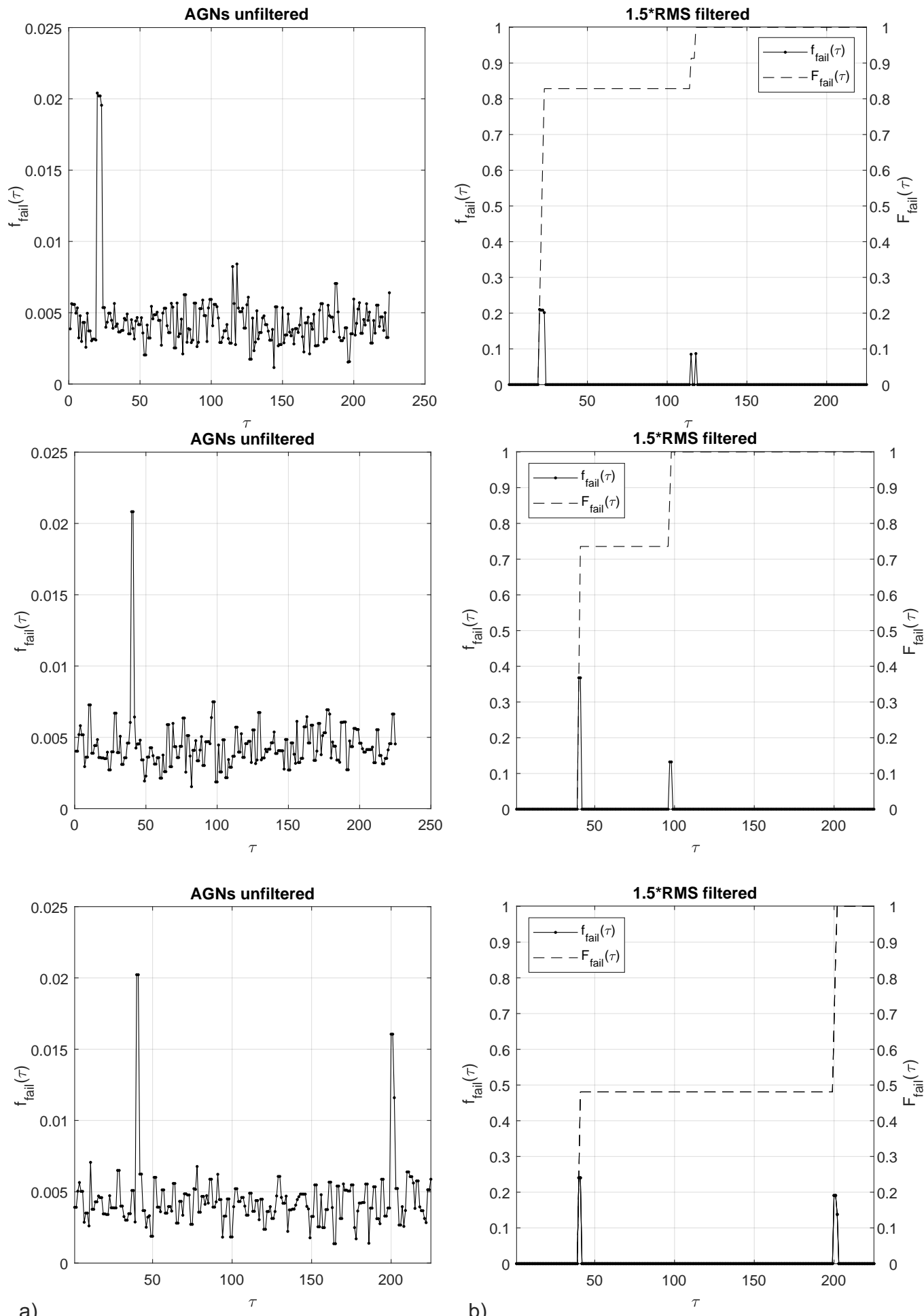

b)

Figure 16. Probability density $f_{\text {fail }}(\tau)$ of a loading profile from a GANs generated probability map (a), probability density $1.5 \times R M S$ filtered $f_{\text {fail }}(\tau)$ and accumulated probability $F_{\text {fail }}(\tau)(\mathbf{b})$. Loading slopes from top to bottom are: $\epsilon_{22} / \epsilon_{11}=2.0, \epsilon_{22} / \epsilon_{11}=3.7$ and $\epsilon_{22} / \epsilon_{11}=4.1$. 
Results highlight the capacity of GANs to provide a failure prediction for a nondeterministic response. Computational economy of the approach can hardly be matched by any numerically-based constitutive model such as FEM or DEM using typical numbers of elements or particles. The avoidance of model collapse, a common issue in GANs, is demonstrated: (Figure 16, first row), where failure probabilities in close-by loading steps 115 and 118 are separated by null failure probability. The approach still suffers from the need to filter the images to eliminate noise; such weakness can possibly be mitigated by the adoption of alternative network architectures and finer hyperparameter tuning.

\section{Discussion}

Results of the different ML algorithms (Table 4) show varying levels of success in reproducing the micro-scale constitutive response. GPR gave good fitting metrics but due to the presence of more than one distribution in the data it was unable to properly reproduce the different failure mechanisms. GPR results can be put down to its own limitations concerning input data. A SOM is able to extract a large number of features from the data given than those features are sufficiently distinguishable. In the present work, SOM failed at properly reproducing some of the scattered regions which are one of the distinctive traits of the micro-scale response. Finally, GANs showed good generation capability for all the failure mechanisms with the advantage of following an unsupervised learning method meaning that no assumptions about input data needed to be made.

Table 4. ML algorithm summary with learning type and neural network depth. A network is said to be deep if it contains two or more hidden layers.

\begin{tabular}{cccc}
\hline Algorithm & Learning & Depth & Typical Application \\
\hline GPR & Supervised & Shallow & Curve fitting \\
SOM & Unsupervised & Shallow & Maps, clustering \\
GANs & Unsupervised & Deep & Fake image generation \\
\hline
\end{tabular}

The depth of a neural network is a measure of its complexity and the power of the network to perform higher level abstractions from the data. Networks' depth in GANs, together with their adversarial training approach are two of the attributes that confer them higher cognitive ability. Nevertheless the typical depth used in image generation applications has been reduced in an application of "Ockham's Razor"; in the XIV century William of Ockham gave the methodological principle (Latin):

"Pluralitas non est ponenda sine neccesitate"

or translated into machine learning "models should be no more complex than is sufficient to explain the data". The principle very much applies to the present work since excessive network complexity in either depth or number of neurons resulted in the "Helvetica scenario" a kind of mode collapse.

Existing surrogates to approximate material constitutive behaviour such as Radial Basis Functions in Surrogate Optimization, among others, cannot reproduce the nondeterminism of the approximated function. The proposed approach trains a generator able to provide an unlimited quantity of constitutive response samples as far as it is fed with random noise. The ability to produce infinite non-repeated samples is of great interest in multiscale approaches needing a material description in each of their Gauss points. GANs can also generate material responses in heterogeneous problems by learning the response distribution of each of the constituents. This approach can bridge the gap between phenomenological approaches where heterogeneity is added using a predefined finite set of material properties and multiscale numerical approaches where the size of the problem is limited by computational cost.

The cost of the proposed method is almost entirely caused by the generation of the training dataset; the 12,000 sample dataset used in the SOM and GANs cases required 
$67 \mathrm{~h}$ of CPU time while the training of the networks only a few minutes. The deployment of the trained generator output in a numerical model has negligible computational cost compared to the solver cost in a typical FEM, this is the same situation as with classical phenomenological expressions. Assuming an already available dataset, the economy of the proposed model is assured in almost all cases compared to a multiscale physics-based approach. In the case of requiring a dataset generation, economy of GANs is advantageous as far as the number of required material point evaluations in the physics-based model surpasses the number of dataset samples. Given the typical mesh refinement used in FEM applications and number of loading steps required in nonlinear problems this scenario is easily met and economy raises quickly to a multifold speedup.

\section{Conclusions}

In the present work a series of Machine Learning paradigms have been proposed to provide the surrogate of a micro-scale model response. The main focus being geomaterials, method and results can be generalized to any material. Main conclusions are listed below:

- Non-determinism is an inherent property of the material mechanical response. It plays a paramount role in the onset end evolution of strain localisation, thus the importance of its reproduction in numerical models.

- Gaussian Process Regression cannot reproduce the micro-scale response because of the presence of multiple distributions rather than a unique random distribution. This is due to the existence of different mechanisms leading to failure.

- Neural net clustering using Self-Organizing Maps can distinguish several of the microscale failure modes but does not succeed at identifying a small group of data points that are an important feature of the response.

- With the adequate network architecture, Generative Adversarial Networks can properly reproduce the micro-scale data including its non-deterministic characteristics. As the learning is totally unsupervised, no assumptions about data distribution are needed.

- The Generator trained in the Generative Adversarial Networks is ready to be deployed in multiscale numerical approaches allowing to, at list partially, avoid the costly numerical micro-computations. The computational economy must allow those multiscale models to compete with classical phenomenological models in terms of cost.

Future research should focus on a full mechanical material description using AGNs i.e., elastic properties, elasto-plastic, visco-plastic and fatigue. Upgrade of AGNs using physics informed networks such as Thermodynamics-based Artificial Neural Networks (TANN) [34]. The $r_{f m}$ coefficient used in the definition of the failure criterion can be enriched with knowledge of the macroscale, this should allow to obtain a holistic multiscale damage model. Data augmentation techniques can help to increase dataset size without requiring additional physics-based simulations. Finally application of the approach in both forward and inverse material design problems and comparison against phenomenological and numerical double-scale models.

Supplementary Materials: The following are available at https:/ /www.mdpi.com/article/10.339 0/ma15030965/s1, Figures S1-S4, GANs Matlab code, micro-scale node numbering. A supporting animation of the GANs generations through training is available at: https://doi.org/10.6084/m9 .figshare.17085446 (posted on 26 November 2021).

Author Contributions: Conceptualization, A.A.; methodology, A.A.; software, A.A.; writingoriginal draft preparation, A.A.; writing-review and editing, A.A. and D.Z.; funding acquisition, A.A. All authors have read and agreed to the published version of the manuscript.

Funding: This research was funded by the Fundamental Research Funds for the Central Universities (No. 2021QNA4024).

Institutional Review Board Statement: Not applicable.

Informed Consent Statement: Not applicable. 
Data Availability Statement: The data presented in this study are openly available within the document and in the supplementary materials.

Conflicts of Interest: The authors declare no conflict of interest. The funders had no role in the design of the study; in the collection, analyses, or interpretation of data; in the writing of the manuscript, or in the decision to publish the results.

\section{Abbreviations}

The following abbreviations are used in this manuscript:

$\begin{array}{ll}\text { AI } & \text { Artificial Intelligence } \\ \text { ML } & \text { Machine Learning } \\ \text { PSO } & \text { Particle Swarm Optimizer } \\ \text { PDE } & \text { Partial Differential Equation } \\ \text { DoF } & \text { Degree of Freedom } \\ \text { GPR } & \text { Gaussian Process Regression } \\ \text { SOM } & \text { Self-Organizing Map } \\ \text { GANs } & \text { Generative Adversarial Networks } \\ \text { LSGANs } & \text { Least Squares Generative Adversarial Networks } \\ \text { DCGANs } & \text { Deep Convolutional Generative Adversarial Networks } \\ \text { WGANs } & \text { Wasserstein Generative Adversarial Networks } \\ \text { TANN } & \text { Thermodynamics-based Artificial Neural Networks }\end{array}$

\section{References}

1. Auriault, J.L. Heterogeneous medium. Is an equivalent macroscopic description possible? Int. J. Eng. Sci. 1991, 29, 785-795. [CrossRef]

2. Argilaga, A.; Papachristos, E. Bounding the Multi-Scale Domain in Numerical Modelling and Meta-Heuristics Optimization: Application to Poroelastic Media with Damageable Cracks. Materials 2021, 14, 3974. [CrossRef] [PubMed]

3. Stefanou, I.; Sulem, J.; Vardoulakis, I. Three-dimensional Cosserat homogenization of masonry structures: Elasticity. Acta Geotech. 2008, 3, 71-83. [CrossRef]

4. Godio, M.; Stefanou, I.; Sab, K.; Sulem, J.; Sakji, S. A limit analysis approach based on Cosserat continuum for the evaluation of the in-plane strength of discrete media: Application to masonry. Eur. J.-Mech.-A/Solids 2017, 66, 168-192. [CrossRef]

5. Scholtès, L.; Donzé, F.V.; Khanal, M. Scale effects on strength of geomaterials, case study: Coal. J. Mech. Phys. Solids 2011, 59, 1131-1146. [CrossRef]

6. Bertrand, F.; Cerfontaine, B.; Collin, F. A fully coupled hydro-mechanical model for the modeling of coalbed methane recovery. $J$. Nat. Gas Sci. Eng. 2017, 46, 307-325. [CrossRef]

7. Godyń, K.; Dutka, B. Sorption and Micro-Scale Strength Properties of Coals Susceptible to Outburst Caused by Changes in Degree of Coalification. Materials 2021, 14, 5807. [CrossRef]

8. Valle, V.; Hedan, S.; Cosenza, P.; Fauchille, A.L.; Berdjane, M. Digital image correlation development for the study of materials including multiple crossing cracks. Exp. Mech. 2015, 55, 379-391. [CrossRef]

9. Ougier-Simonin, A.; Renard, F.; Boehm, C.; Vidal-Gilbert, S. Microfracturing and microporosity in shales. Earth-Sci. Rev. 2016, 162, 198-226. [CrossRef]

10. Arson, C. Micro-macro mechanics of damage and healing in rocks. Open Geomech. 2020, 2, 1-41. [CrossRef]

11. Cartwright-Taylor, A.; Mangriotis, M.D.; Main, I.; Butler, I.; Fusseis, F.; Ling, M.; Andò, E.; Curtis, A.; Bell, A.; Crippen, A.; et al. Seismic events miss important grain-scale mechanisms governed by kinematics during shear failure of porous rock. Nat. Portf. 2022, 2693-5015. [CrossRef]

12. Meier, H.; Steinmann, P.; Kuhl, E. Towards multiscale computation of confined granular media-Contact forces, stresses and tangent operators. Tech. Mech. 1996, 1, 77-88.

13. Nitka, M.; Combe, G.; Dascalu, C.; Desrues, J. Two-scale modeling of granular materials: A DEM-FEM approach. Granul. Matter 2011, 13, 277-281. [CrossRef]

14. de Souza Neto, E.; Blanco, P.; Sánchez, P.; Feijóo, R. An RVE-based multiscale theory of solids with micro-scale inertia and body force effects. Mech. Mater. 2015, 80, 136-144. [CrossRef]

15. van den Eijnden, B.; Bésuelle, P.; Chambon, R.; Collin, F. A FE2 modelling approach to hydromechanical coupling in crackinginduced localization problems. Int. J. Solids Struct. 2016, 97-98, 475-488. [CrossRef]

16. Desrues, J.; Argilaga, A.; Caillerie, D.; Combe, G.; Nguyen, T.; Richefeu, V.; Dal Pont, S. From discrete to continuum modelling of boundary value problems in geomechanics: An integrated FEM-DEM approach. Int. J. Numer. Anal. Methods Geomech. 2019, 43, 919-955. [CrossRef] 
17. Pardoen, B.; Bésuelle, P.; Dal Pont, S.; Cosenza, P.; Desrues, J. Accounting for Small-Scale Heterogeneity and Variability of Clay Rock in Homogenised Numerical Micromechanical Response and Microcracking. Rock Mech. Rock Eng. 2020, 53, 2727-2746. [CrossRef]

18. Guo, N.; Yang, Z.; Yuan, W.H.; Zhao, J. A coupled SPFEM/DEM approach for multiscale modeling of large-deformation geomechanical problems. Int. J. Numer. Anal. Methods Geomech. 2020, 45, 648-667. [CrossRef]

19. Papanicolau, G.; Bensoussan, A.; Lions, J.L. Asymptotic Analysis for Periodic Structures; Elsevier: Amsterdam, The Netherlands, 1978.

20. Sánchez-Palencia, E. Non-Homogeneous Media and Vibration Theory; Springer: Berlin, Germany, $1980 ;$ Volume 127.

21. Arbogast, T.; Douglas, J., Jr.; Hornung, U. Derivation of the double porosity model of single phase flow via homogenization theory. SIAM J. Math. Anal. 1990, 21, 823-836. [CrossRef]

22. Sridhar, A.; Kouznetsova, V.; Geers, M. A general multiscale framework for the emergent effective elastodynamics of metamaterials. J. Mech. Phys. Solids 2017, 111, 411-433. [CrossRef]

23. Waseem, A.; Heuzé, T.; Stainier, L.; Geers, M.; Kouznetsova, V. Model reduction in computational homogenization for transient heat conduction. Comput. Mech. 2020, 65, 249-266. [CrossRef]

24. Auriault, J.L. Heterogeneous periodic and random media. Are the equivalent macroscopic descriptions similar? Int. J. Eng. Sci. 2011, 49, 806-808. [CrossRef]

25. Argilaga, A.; Papachristos, E.; Caillerie, D.; Dal Pont, S. Homogenization of a cracked saturated porous medium: Theoretical aspects and numerical implementation. Int. J. Solids Struct. 2016, 94-95, 222-237. [CrossRef]

26. Chen, C.T.; Gu, G.X. Effect of Constituent Materials on Composite Performance: Exploring Design Strategies via Machine Learning. Adv. Theory Simul. 2019, 2, 1900056. [CrossRef]

27. Liu, X.; Athanasiou, C.E.; Padture, N.P.; Sheldon, B.W.; Gao, H. A machine learning approach to fracture mechanics problems. Acta Mater. 2020, 190, 105-112. [CrossRef]

28. Hanakata, P.Z.; Cubuk, E.D.; Campbell, D.K.; Park, H.S. Forward and inverse design of kirigami via supervised autoencoder. Phys. Rev. Res. 2020, 2, 042006. [CrossRef]

29. Wang, K.; Sun, W. A multiscale multi-permeability poroplasticity model linked by recursive homogenizations and deep learning Comput. Methods Appl. Mech. Eng. 2018, 334, 337-380. [CrossRef]

30. Liu, Z.; Wu, C. Exploring the 3D architectures of deep material network in data-driven multiscale mechanics. J. Mech. Phys. Solids 2019, 127, 20-46. [CrossRef]

31. Logarzo, H.J.; Capuano, G.; Rimoli, J.J. Smart constitutive laws: Inelastic homogenization through machine learning. Comput. Methods Appl. Mech. Eng. 2021, 373, 113482. [CrossRef]

32. Jang, D.P.; Fazily, P.; Yoon, J.W. Machine learning-based constitutive model for J2-plasticity. Int. J. Plast. 2021, 138, 102919. [CrossRef]

33. Vlassis, N.N.; Sun, W. Sobolev training of thermodynamic-informed neural networks for interpretable elasto-plasticity models with level set hardening. Comput. Methods Appl. Mech. Eng. 2021, 377, 113695. [CrossRef]

34. Masi, F.; Stefanou, I.; Vannucci, P.; Maffi-Berthier, V. Thermodynamics-based Artificial Neural Networks for constitutive modeling. J. Mech. Phys. Solids 2021, 147, 104277. [CrossRef]

35. Kim, D.W.; Lim, J.H.; Lee, S. Prediction and validation of the transverse mechanical behavior of unidirectional composites considering interfacial debonding through convolutional neural networks. Compos. Part Eng. 2021, 225, 109314. [CrossRef]

36. Kim, Y.; Kim, Y.; Yang, C.; Park, K.; Gu, G.; Ryu, S. Deep learning framework for material design space exploration using active transfer learning and data augmentation. NPJ Comput. Mater. 2021, 7, 140. [CrossRef]

37. Ashank; Chakravarty, S.; Garg, P.; Kumar, A.; Agrawal, M.; Agnihotri, P.K. Deep Neural Networks Based Predictive-Generative Framework for Designing Composite Materials. 2021. Available online: http:/ / www.lanl.gov/abs/2105.01384 (accessed on 1 November 2021).

38. Papachristos, E.; Stefanou, I. Controlling earthquake-like instabilities using artificial intelligence. arXiv 2021, arXiv:2104.13180.

39. Maizir, H.; Gofar, N.; Kassim, K. Artificial Neural Network Model for Prediction of Bearing Capacity of Driven Pile. J. Tek. Sipil Inst. Teknol. Bdg. 2015, 22, 49-56.

40. Benbouras, M.A.; Petrişor, A.I.; Zedira, H.; Ghelani, L.; Lefilef, L. Forecasting the Bearing Capacity of the Driven Piles Using Advanced Machine-Learning Techniques. Appl. Sci. 2021, 11, 908. [CrossRef]

41. Jahed Armaghani, D.; Harandizadeh, H.; Momeni, E.; Maizir, H.; Zhou, J. An optimized system of GMDH-ANFIS predictive model by ICA for estimating pile bearing capacity. Artif. Intell. Rev. 2021, 54, 1-38. [CrossRef]

42. Omar, M.; Che Mamat, R.; Abdul Rasam, A.R.; Ramli, A.; Samad, A. Artificial intelligence application for predicting slope stability on soft ground: A comparative study. Int. J. Adv. Technol. Eng. Explor. 2021, 8, 362-370. [CrossRef]

43. Chambon, R.; Caillerie, D.; Viggiani, G. Loss of uniqueness and bifurcation vs instability: Some remarks. Rev. Fr. Génie Civ. 2004, 8,517-535. [CrossRef]

44. Hill, R. Acceleration waves in solids. J. Mech. Phys. Solids 1962, 10, 1-16. [CrossRef]

45. Rice, J.R. The Localization of Plastic Deformation; Division of Engineering, Brown University: Providence, RI, USA, 1976.

46. Pietruszczak, S.; Mroz, Z. Finite element analysis of deformation of strain-softening materials. Int. J. Numer. Methods Eng. 1981, 17, 327-334. [CrossRef]

47. Sluys, L.; De Borst, R.; Mühlhaus, H.B. Wave propagation, localization and dispersion in a gradient-dependent medium. Int. J. Solids Struct. 1993, 30, 1153-1171. [CrossRef] 
48. Argilaga, A.; Desrues, J.; Dal Pont, S.; Combe, G.; Caillerie, D. FEM $\times$ DEM multiscale modeling: Model performance enhancement from Newton strategy to element loop parallelization. Int. J. Numer. Methods Eng. 2018, 114, 47-65. [CrossRef]

49. Eringen, A.C. Nonlocal polar elastic continua. Int. J. Eng. Sci. 1972, 10, 1-16. [CrossRef]

50. Mindlin, R.D. Second gradient of strain and surface-tension in linear elasticity. Int. J. Solids Struct. 1965, 1, 417-438. [CrossRef]

51. Germain, P. La méthode des puissances virtuelles en mécanique des milieux continus. J. Mec. 1973, 12, $236-274$.

52. De Borst, R.; Mühlhaus, H.B. Gradient-dependent plasticity: Formulation and algorithmic aspects. Int. J. Numer. Methods Eng. 1992, 35, 521-539. [CrossRef]

53. Pamin, J.K. Gradient-Dependent Plasticity in Numerical Simulation of Localization Phenomena; TU Delft, Delft University of Technology: Amsterdam, The Netherlands, 1994.

54. Peerlings, R.H.; de Borst, R.; Brekelmans, W.M.; de Vree, J. Gradient enhanced damage for quasi-brittle materials. Int. J. Numer. Methods Eng. 1996, 39, 3391-3403. [CrossRef]

55. Chambon, R.; Caillerie, D.; El Hassan, N. One-dimensional localisation studied with a second grade model. Eur. J.-Mech.-A/Solids 1998, 17, 637-656. [CrossRef]

56. Matsushima, T.; Chambon, R.; Caillerie, D. Second gradient models as a particular case of microstructured models: A large strain finite elements analysis. C. R. l'Académie Sci. IIB-Mech. 2000, 328, 179-186. [CrossRef]

57. Chambon, R.; Caillerie, D.; Matsuchima, T. Plastic continuum with microstructure, local second gradient theories for geomaterials: Localization studies. Int. J. Solids Struct. 2001, 38, 8503-8527. [CrossRef]

58. Yang, Y.; Misra, A. Micromechanics based second gradient continuum theory for shear band modeling in cohesive granular materials following damage elasticity. Int. J. Solids Struct. 2012, 49, 2500-2514. [CrossRef]

59. Collin, F.; Chambon, R.; Charlier, R. A finite element method for poro mechanical modelling of geotechnical problems using local second gradient models. Int. J. Numer. Methods Eng. 2006, 65, 1749-1772. [CrossRef]

60. Marinelli, F. Comportement Couplé des Géomatériaux: Deus Approches de Módelisation Numérique. Ph.D. Thesis, Communauté Université Grenoble Alpes, Saint-Martin-d'Hères, France, 2013.

61. Jouan, G.; Kotronis, P.; Collin, F. Using a second gradient model to simulate the behaviour of concrete structural elements. Finite Elem. Anal. Des. 2014, 90, 50-60. [CrossRef]

62. Salehnia, F.; Collin, F.; Li, X.L.; Dizier, A.; Sillen, X.; Charlier, R. Coupled modeling of Excavation Damaged Zone in Boom clay: Strain localization in rock and distribution of contact pressure on the gallery's lining. Comput. Geotech. 2015, 69, 396-410. [CrossRef]

63. Sieffert, Y.; Al Holo, S.; Chambon, R. Loss of uniqueness of numerical solutions of the borehole problem modelled with enhanced media. Int. J. Solids Struct. 2009, 46, 3173-3197. [CrossRef]

64. Desrues, J.; Argilaga, A.; Pont, S.D.; Combe, G.; Caillerie, D.; Nguyen, T.K. Restoring Mesh Independency in FEM-DEM Multi-Scale Modelling of Strain Localization Using Second Gradient Regularization; Springer Series in Geomechanics and Geoengineering; Springer International Publishing: Cham, Switzerland, 2017; pp. 453-457.

65. Guo, S.; Qi, S.; Zou, Y.; Zheng, B. Numerical Studies on the Failure Process of Heterogeneous Brittle Rocks or Rock-Like Materials under Uniaxial Compression. Materials 2017, 10, 378. [CrossRef]

66. Shahin, G.; Cil, M.; Buscarnera, G. Assessment of statistical homogeneity in chemically treated granular materials. Geotech. Lett. 2018, 8, 32-39. [CrossRef]

67. Pardoen, B.; Dal Pont, S.; Desrues, J.; Bésuelle, P.; Prêt, D.; Cosenza, P. Heterogeneity and Variability of Clay Rock Microstructure in a Hydro-Mechanical Double Scale FEM $\times$ FEM Analysis. In Micro to MACRO Mathematical Modelling in Soil Mechanics; Giovine, P., Mariano, P.M., Mortara, G., Eds.; Springer International Publishing: Cham, Switzerland, 2018; pp. 247-256. [CrossRef]

68. Pardoen, B.; Collin, F. Modelling the influence of strain localisation and viscosity on the behaviour of underground drifts drilled in claystone. Comput. Geotech. 2016, 85, 351-367. [CrossRef]

69. Shahin, G.; Desrues, J.; Pont, S.D.; Combe, G.; Argilaga, A. A study of the influence of REV variability in double-scale FEM $\times$ DEM analysis. Int. J. Numer. Methods Eng. 2016, 107, 882-900. [CrossRef]

70. Cheng, X.; Tang, C.; Zhuang, D. A finite-strain viscoelastic-damage numerical model for time-dependent failure and instability of rocks. Comput. Geotech. 2022, 143, 104596. [CrossRef]

71. Andò, E.; Viggiani, G.; Hall, S.; Desrues, J. Experimental micro-mechanics of granular media studied by X-ray tomography: Recent results and challenges. Geotech. Lett. 2013, 3, 142-146. [CrossRef]

72. Couture, C.; Bésuelle, P. Diffuse and localized deformation of a porous Vosges sandstone in true triaxial conditions. E3S Web Conf. 2019, 92, 06007. [CrossRef]

73. Pardoen, B.; Bésuelle, P.; Dal Pont, S.; Cosenza, P.; Desrues, J. Effect of Claystone Small-Scale Characteristics on the Variability of Micromechanical Response and on Microcracking Modelling; Challenges and Innovations in Geomechanics; Barla, M., Di Donna, A., Sterpi, D., Eds.; Springer International Publishing: Cham, Switzerland, 2021; pp. 522-530.

74. Tudisco, E.; Vitone, C.; Mondello, C.; Viggiani, G.; Athanasopoulos, S.; Hall, S.; Cotecchia, F. Localised strain in fissured clays: The combined effect of fissure orientation and confining pressure. Acta Geotech. 2021, 1-9. [CrossRef]

75. Royer, P.; Cherblanc, F. Homogenisation of advective-diffusive transport in poroelastic media. Mech. Res. Commun. 2010, 37, 133-136. [CrossRef]

76. Auriault, J.L. Transport in porous media: Upscaling by multiscale asymptotic expansions. In Applied Micromechanics of Porous Materials; Springer: Vienna, Austria, 2005; pp. 3-56. 
77. Caillerie, D. Thin and periodic plates. Math. Meth. Appl. Sci. 1984, 6, 159-191. [CrossRef]

78. Dascalu, C.; Bilbie, G.; Agiasofitou, E. Damage and size effects in elastic solids: A homogenization approach. Int. J. Solids Struct. 2008, 45, 409-430. [CrossRef]

79. Marinelli, F.; Sieffert, Y.; Chambon, R. Hydromechanical modeling of an initial boundary value problem: Studies of nonuniqueness with a second gradient continuum. Int. J. Solids Struct. 2015, 54, 238-257. [CrossRef]

80. Nova, R. Controllability of the incremental response of soil specimens subjected to arbitrary loading programmes. J. Mech. Behav. Mater. 1994, 5, 193-202. [CrossRef]

81. Rasmussen, C.E. Gaussian Processes in Machine Learning. In Advanced Lectures on Machine Learning: ML Summer Schools 2003, Canberra, Australia, 2-14 February 2003, Tübingen, Germany, 4-16 August 2003, Revised Lectures; Bousquet, O., von Luxburg, U., Rätsch, G., Eds.; Springer: Berlin/Heidelberg, Germany, 2004; pp. 63-71. [CrossRef]

82. Qian, J.; Nguyen, N.P.; Oya, Y.; Kikugawa, G.; Okabe, T.; Huang, Y.; Ohuchi, F.S. Introducing self-organized maps (SOM) as a visualization tool for materials research and education. Results Mater. 2019, 4, 100020. [CrossRef]

83. Goodfellow, I.J. NIPS 2016 Tutorial: Generative Adversarial Networks. CoRR 2017. Available online: http://www.lanl.gov/abs/ 1701.00160 (accessed on 5 November 2021).

84. Mao, X.; Li, Q.; Xie, H.; Lau, R.Y.K.; Wang, Z.; Smolley, S.P. Least Squares Generative Adversarial Networks. 2017. Available online: http:/ / www.lanl.gov/abs/1611.04076 (accessed on 5 November 2021).

85. Radford, A.; Metz, L.; Chintala, S. Unsupervised Representation Learning with Deep Convolutional Generative Adversarial Networks. 2016. Available online: http:/ / www.lanl.gov/abs/1511.06434 (accessed on 5 November 2021).

86. Arjovsky, M.; Chintala, S.; Bottou, L. Wasserstein Generative Adversarial Networks. In Proceedings of the 34th International Conference on Machine Learning-Volume 70, Sydney, NSW, Australia, 6-11 August 2017; pp. 214-223.

87. Metz, L.; Poole, B.; Pfau, D.; Sohl-Dickstein, J. Unrolled Generative Adversarial Networks. 2017. Available online: http: / / www.lanl.gov/abs/1611.02163 (accessed on 5 November 2021). 